\title{
Physics-based Modeling of an Anthropomimetic Robot
}

\author{
Steffen Wittmeier, Michael Jäntsch, Konstantinos Dalamagkidis and Alois Knoll
}

\begin{abstract}
The control of tendon-driven robots using techniques from traditional robotics remains a very challenging task that has been so far only successfully achieved for small-scale setups comprising exclusively revolute joints $[1,2]$. Hence, we propose a fundamentally different approach. Instead of deriving an analytical robot model using either the Newton-Euler or Lagrangian formulation we suggest to employ physics-based simulation engines to simulate the peculiar dynamics of this emerging class of robots and to use the simulated robot model as an internal model for robot control [3].

In this paper, we present the reverse-engineered derivation of a detailed physics-based model of an anthropomimetic robot implemented on CALIPER [4], a simulation framework developed within the EU-funded project ЕССЕRовот [5]. The model comprises an accurate model of the skeleton derived from laser scan data, as well as of artificial ligaments and muscles. The individual sub-models are validated separately against measurements and the successful integration of all sub-models is demonstrated by executing a limb movement which requires the parallel control of multiple muscles.
\end{abstract}

\section{INTRODUCTION}

Due to their versatile application capabilities, computer simulations have become an indispensable tool for engineers and researchers during the last decades. Typical areas of application are the modeling of natural phenomena [6], of economic processes [7] and of products under development for design optimization [8].

In robotics, as well, simulations have become an important tool that is mainly used offline for controller design and optimization as cheap and safe substitutes of the real hardware [9]. For traditional robots, a simulation model can be derived analytically using either the Newton-Euler or Lagrangian formulation. For tendon-driven robots, however, a similar approach is not feasible due to the sheer complexity of the resulting analytical model, especially when collisions between the tendons and the mechanical structure of the robot occur. Therefore, the precise control of tendon-driven robots using techniques from traditional robotics remains a very challenging task that has been so far only successfully achieved for small-scale setups comprising only revolute joints $[1,2]$. Hence, to tackle this problem we propose a fundamentally different approach. Instead of analytically deriving a tendondriven robot model we suggest to utilize a physics-based simulation engine such as PhysX [10] or Bullet Physics [11] to simulate the complicated dynamics of tendon-driven robots

S. Wittmeier, M. Jäntsch, K. Dalamagkidis and A. Knoll are with the Chair of Robotics and Embedded Systems, Faculty of Informatics, Technische Universität München, Munich, Germany, Correspondence should be addressed to S. Wittmeier (wittmeis@in.tum.de)

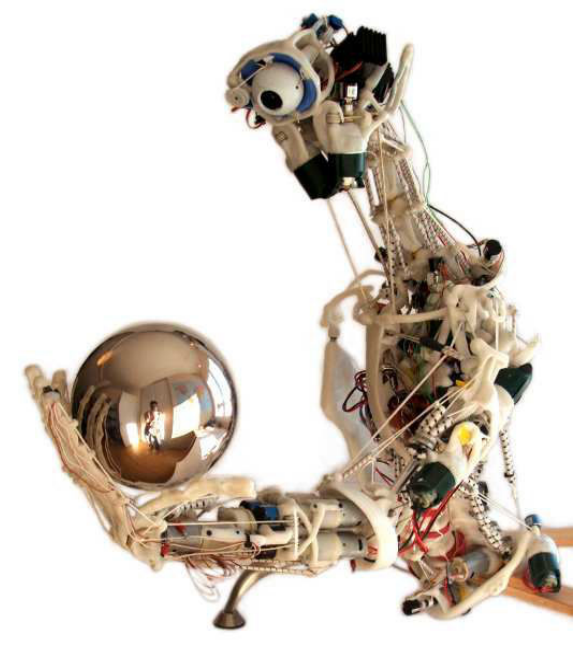

Fig. 1. ECCE-I PROTOTYPE. Prototype of the anthropomimetic [12] robot ECCE-I developed within the EU-funded project ECCEROBOT [5, 13]. The skeleton is hand-crafted using polymorph - a caprolactone polymer-which can easily be molded. The human muscles are imitated by elastic, tendondriven actuators comprising a DC motor and a gearbox in series with a kite line and shock cord that function as the muscle fibers and tendon, respectively.

and to employ this simulation model as an internal model for robot control [3].

In this paper we present our efforts in deriving an accurate physics-based model of a simplified test rig of the anthropomimetic [12] robot ECCE-I which has been developed within the EU-funded project Embodied Cognition In A Compliantly Engineered Robot (ЕССЕROBOT, see Fig.1) $[5,13]$. The physics-based model has been implemented on CALIPER [4], a simulation framework based on Bullet Physics and developed within the ЕССЕRОвот project.

Section II describes the anthropomimetic robot platform, followed by the derivation of the skeleton CAD and physics model of this robot in Section III. Sections IV and V cover the derivation of the ligament and muscle model, respectively. The sensor models are presented in Section VI whereas Section VII presents the implementation of low level muscle control. Conclusions and future work prospects are covered in Section VIII.

\section{The Anthropomimetic Robot Platform}

There are many humanoid robotic projects being undertaken around the world. However, most of them focus on adopting the morphological appearance of humans to facilitate humanrobot interaction [14, 15]. Moreover, most of these robots 


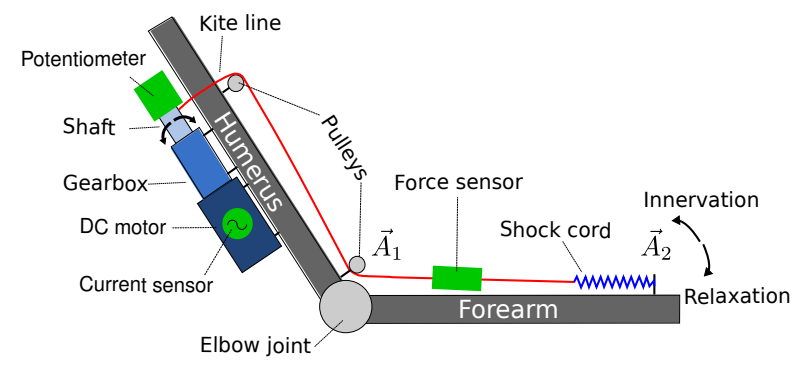

Fig. 2. Eccerobot Tendon-Driven Muscle. The Eccerobot muscle comprises a DC motor, a gearbox and kite line in series with an elastic shock cord. By coiling the kite line around the gearbox shaft the muscle exerts force at the attachment points $\vec{A}_{1}$ and $\vec{A}_{2}$. For proprioception, each muscle is equipped with a potentiometer as well as a force and current sensor.

are actuated and controlled using sophisticated engineering techniques of extremely high standard which, of course, results in an impressive performance for the tasks that they are designed for (e.g. [14]). But these robots are still far from capturing the distinct dynamics and impressive adaptability to changing environments of humans. Hence, the EU-funded project ЕССЕRОВОт takes a fundamentally different, namely anthropomimetic [12], approach. Instead of only imitating the morphological appearance of humans it tries to also mimic the internal structures of humans: bones, joints, muscles and tendons.

Currently, three ECCEROBOTS exist: (i) ECCE-I (see Fig. 1 and [13]), (ii) the ECCEROBot Design Study (EDS) [13] and (iii) a simplified test rig (see Fig. 3A). The skeletons of all prototypes are hand-crafted using polymorph-a caprolactone polymer-which can easily be molded at a temperature of only $60^{\circ} \mathrm{C}$. Typical robotic actuators are very different from muscles. Hence, instead of employing high-precision stiff joint actuators, the ECCEROBOTS use compliant tendon-driven actuators as muscle equivalents. Each actuator consists of a DC motor and a gearbox in series with a kite line and a shock cord that function as the muscle fibers and tendon, respectively (see Fig. 2). By coiling the kite line around the gearbox shaft, the artificial muscle can be either innervated or relaxed depending on the direction of rotation. Even though humans have $\sim 650$ different muscles, most of these muscles are fairly stereotyped. Hence, only the most important muscles are replicated and the attachment points are chosen accordingly to replicate humanlike movement dynamics. For proprioception-the sense of static positions and movements of the limbs of the body [16]each actuator is equipped with: (i) a potentiometer to measure the position of the gearbox shaft, (ii) a strain gauge attached to the kite line for force sensing, providing an estimate of the extension of the shock cord and (iii) a current sensor within the electronic control units (ECUs) for motor control [17] (see Fig. 2). By fusing the information from these sensors the total length of the artificial muscle can be calculated which makes it subsequently possible to estimate the current body pose.

ECCE-I and the EDS consist of more than 40 powered degrees of freedom. Hence, to reduce the complexity for the first experiments and for evaluating possible shoulder
A

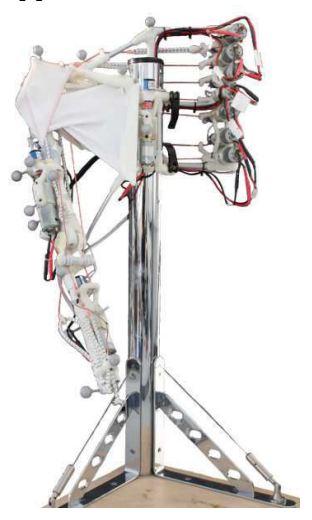

C

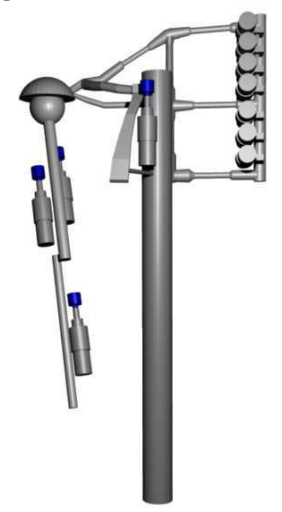

B

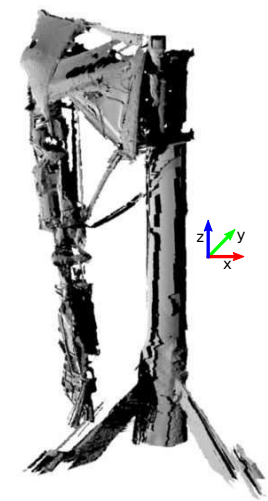

D

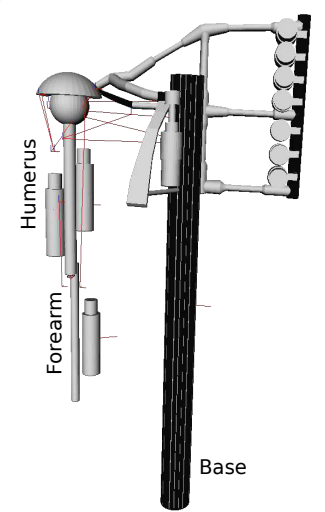

Fig. 3. Skeleton Model. A: Eccerobot test rig. The test rig has a spherical shoulder joint, a revolute elbow joint and 11 tendon-driven muscles. B: Laser scan CAD model registered from 25 individual scans. C: Static CAD model. D: Dynamic, physics-based model rendered by the simulation framework CALIPER [4].

designs the simplified test rig has been manufactured by the ECCEROBOT consortium (see Fig. 3A). This test rig comprises a spherical joint as shoulder and a revolute joint as elbow joint, as well as 11 powered degrees of freedom-2 for the elbow, 8 for the shoulder and 1 bi-articular actuator affecting both shoulder and elbow joint angles. Due to its reduced complexity this test rig has proven to be highly beneficial for investigating possible control algorithms and hence, it is the platform modeled in this paper. Once the modeling techniques employed in this work have proven to be successful, a detailed model of the ECCE-I successor ECCE-II, that will be available soon, will be derived based on the lessons learned.

\section{SKELETON MODEL}

Due to the hand-crafted manufacturing of the bones, the impossibility of dismantling/reassembling individual components and the unavailability of CAD design data, the derivation of an accurate skeleton model is a highly difficult task. This is further complicated by the bone shapes which do not match common CAD shape primitives such as cylinders or boxes. For the physics-engine, however, it is necessary to approximate these hand-crafted shapes by shape primitives to achieve fast collision detection and good overall simulation performance. 


\section{A. Static Model}

The static CAD skeleton model was derived in two iterations using the open-source CAD tool Blender. In the first iteration we used true to scale photographs to define a first CAD approximation of the skeleton structure. However, this approach suffered from two major drawbacks: (i) attachments, such as DC motors or potentiometers were difficult to accurately position and orient in 3D and (ii) size-inaccuracies due to the parallax error. To cope with these problems we created a total of 25 laser scans of the entire test-rig (see Fig. 3B) and refined the CAD model of the first iteration. However, high-precision scan results could not be obtained of all parts of the test rig due to the hollow structures and floating cables. The resulting noisy scan results also imposed additional challenges on the scan registration but satisfactory results could be achieved by constraining the registration routine so that the individual scans are only rotated around their z-axis (see Fig. 3B). By merging the rough model of the first iteration with the scan data it was possible to accurately position motors and potentiometers within the CAD model (see Fig. 3C). This was necessary as the motors have a high impact on the model dynamics due to their rather high weight compared to the polymorph bones.

\section{B. Dynamic Model}

This static Blender CAD model was then converted to a dynamic model within the Bullet Physics engine (see Fig. 3D). As the used simulation framework CALIPER is capable of importing CAD models using the open-standard XML schema COLLADA, the model was first exported using the COLLADA exporter plugin included in Blender and subsequently modified manually.

1) Rigid Bodies: Within Bullet Physics each moving entity is represented by a rigid body associated with a mass, a center of mass, a shape for collision detection, and an inertia tensor calculated based on the mass and the shape primitive assigned. Hence, the static CAD model was decomposed into separate rigid bodies for the forearm, the humerus and the base (see Fig. 3D). However, assigning a single collision shape to each of these three rigid bodies, such as a bounding cylinder, would result in erroneous collision detection and in an inaccurate inertia tensor and therefore, compound collision shapes with the bone as root shape were used. Here, each entity (bones, actuators and potentiometers) was approximated by a cylinder associated with a mass and a transform defined in the frame of reference of the root shape. This information was subsequently used to calculate the total inertia and the center of mass of the compound rigid body. The mass of the actuator component (DC motor, gearbox and gearbox shaft), as well as of the potentiometer could be measured. However, for the assembled bones this was not possible. Here, the mass was calculated based on the volume of the bone and the polymorph density $\left(1.10 \mathrm{~g} / \mathrm{cm}^{3}\right)$.

2) Constraints: The movement of the individual rigid bodies is limited by constraints in the shoulder (spherical) and elbow (revolute joint). These holonomic constraint types are already part of Bullet Physics and were parametrized accordingly to fit, for instance, the joint angle limits of the test rig. However, preliminary simulations revealed that the simulation model was highly underdamped. Hence, viscous, as well as Coulomb friction models were implemented for these Bullet Physics constraints (see also Fig. 4A). Bullet Physics uses a velocity-based formulation for the constraint equations which bears some advantages over an acceleration-based approach as described in [18]. In this velocity-based constraint representation, constraint motors can be implemented that apply torques to drive the joint with a desired velocity $v_{d}$. These motors can be used to simulate the effects of Coulomb friction (with the simplification that the static and kinetic modalities of the Coulomb friction are equal) by setting $v_{d}=0$ and by defining a maximum torque $\tau_{\max }$. Then, if $v_{d}$ is exceeded, the joint motor will apply $\tau_{\max }$ to bring the velocity back to $v_{d}$ and, if $v_{d}$ is reached, any torque between 0 and $\tau_{\max }$ to keep the desired speed $v_{d}$. The effects of viscous friction were modeled by applying an angular impulse $\vec{I}$ to the attached rigid bodies of a constraint during each simulation step equal to:

$$
\vec{I}=c_{V} \vec{\omega}_{C} \triangle t
$$

where $c_{V}$ is the viscous friction coefficient, $\vec{\omega}_{C}$ is the constraint velocity (relative angular velocity of the two attached rigid bodies) and $\Delta t$ is the simulation step-size.

\section{LIGAMENT MODEL}

In anatomy, a ligament denotes passive fibers attached to two neighboring bones that constrain the joint motion. For the ECCEROBOTS, ligament equivalents are used, for instance, to prevent the shoulder from dislocating. These artificial ligaments comprise either only a kite line (stiff ligaments) or a kite line in series with an elastic shock cord (compliant ligaments) attached to two neighboring artificial bones. These two ligament variants have been modeled and implemented for Bullet Physics using (i) a virtual kite line with no representation as a physical entity within the physics engine and (ii) a physical kite line simulated using soft-body dynamics. For compliant ligaments an additional virtual shock cord has been implemented.

Experimental analysis of the properties of the ECCEROBOT shock cord have shown that it can be accurately approximated by a linear spring-damper model within an expansion range of $80 \%$ of the resting length:

$$
L_{S_{\triangle}}=L_{S}-L_{S_{R}} \leq 0.8 \cdot L_{S_{R}}
$$

where $L_{S_{\triangle}}$ is the shock cord expansion, $L_{S}$ is the current shock cord length and $L_{S_{R}}$ is the shock cord resting length. Hence, the ligament force $F_{L}$ exerted by the shock cord is equal to:

$$
\begin{aligned}
F_{L} & =F_{S}+F_{D} \\
& =k L_{S_{\triangle}}+D \frac{d}{d t} L_{S_{\triangle}}
\end{aligned}
$$

with $F_{L} \geq 0$ 
A

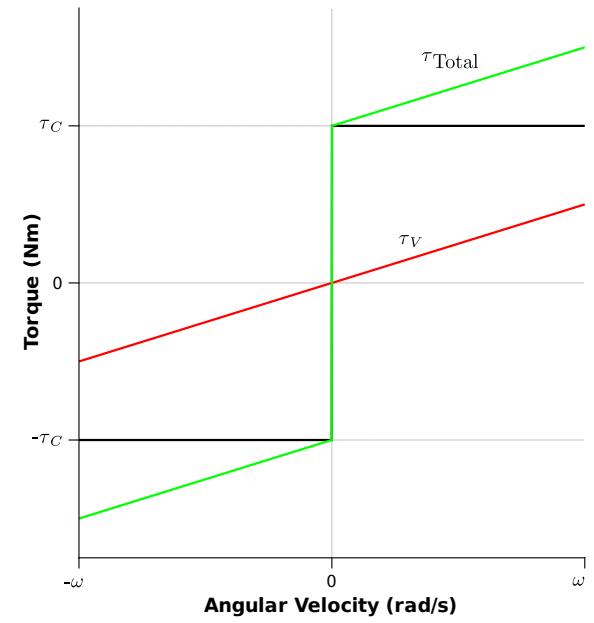

B

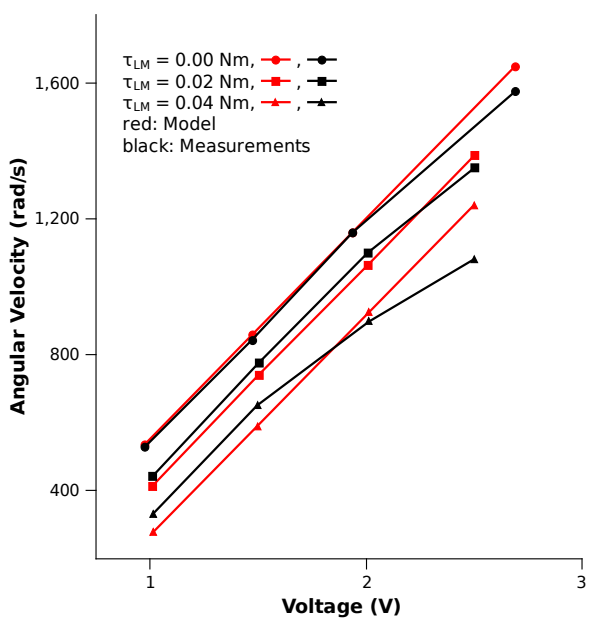

C

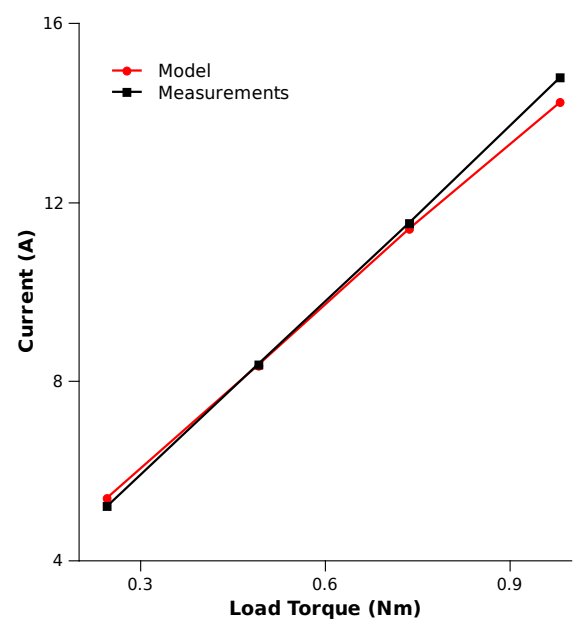

Fig. 4. Friction, DC motor and Gearbox Models. A: Viscous $\tau_{V}$ and Coulomb friction $\tau_{C}$ were implemented for the joints (spherical and revolute) to increase damping and hence the stability of the simulation, as well as for the motor and gearbox models to close the simulation-reality gap of the models. For model simplifications, the static and kinetic modalities of the Coulomb friction were considered to be equal. B: Motor model fitting. C: Motor \& gearbox model fitting.

where $F_{S}$ is the spring force, $k$ is the spring constant, $F_{D}$ is the damper force and $D$ is the damping constant. As ligaments can only pull and not push the additional condition of (4) is required. The length $L_{S}$ of the shock cord can be calculated as follows:

$$
L_{S}=L_{L_{v / p}}-L_{K}
$$

where $L_{L_{v}}$ and $L_{L_{p}}$ are the ligament length of the virtual and physical ligament implementation, respectively, and $L_{K}$ is the kite line length. For the virtual kite line implementation, no collisions between the kite line and the skeleton are considered and hence $L_{L_{v}}$ is equal to the Euclidean distance between the two attachment points $\vec{A}_{1}$ and $\vec{A}_{2}$ (see Fig. 2):

$$
L_{L_{v}}=\left\|\overrightarrow{A_{1}}-\vec{A}_{2}\right\|
$$

For the soft body kite line implementation $L_{L_{p}}$ is equal to:

$$
L_{L_{p}}=\left(\sum_{i=1}^{n}\left\|\vec{N}_{i}-\vec{N}_{i-1}\right\|\right)+\left\|\vec{A}_{2}-\vec{N}_{n}\right\|
$$

where $\vec{N}_{i}$ are the $3 \mathrm{D}$ coordinates of the $i$ th soft body node and $\vec{N}_{0} \equiv \vec{A}_{1}$.

While computationally highly efficient, the virtual kite line implementation suffers from two main problems: (i) no collisions between the skeleton and the kite line are considered, resulting in erroneous force vectors for some configurations and (ii) stiff ligaments can only be approximated using high spring constants. The physical kite line implementation, however, can cope with these problems but introduces new ones due to the high computational load and simulation instabilities resulting from the collisions between the kite line and the skeleton.

\section{Muscle Model}

As presented in Section II, the ECCEROBOT muscles comprise a DC motor and gearbox in series with kite line and shock cord. For the muscle model, the passive kite line and shock cord models presented in the previous section were combined with a DC motor and gearbox model to simulate the shortening/lengthening of the kite line which results in an expansion of the shock cord and hence in a force applied to the two muscle anchor points $\overrightarrow{A_{1}}$ and $\overrightarrow{A_{2}}$ (see Fig. 2).

\section{A. DC Motor Model}

As the ECCEROBOTS use mass-produced screwdriver DC motors to reduce production costs, no data sheets of the motors are available. Hence, manual measurements were conducted to analyze the dynamics of the motor and to derive an accurate mathematical model. Therefore, the electric current and angular velocity of the motor shaft was recorded for different load torques and input voltages. The fitted model is based on a standard DC motor model [19] equal to:

$$
\begin{aligned}
V & =L \frac{d i}{d t}+R i+K_{E} \cdot \omega_{M} \\
\tau_{L_{M}} & =K_{T} \cdot i-J_{M} \frac{d \omega_{M}}{d t}-\mu_{M} \omega_{M}-\tau_{C_{M}}
\end{aligned}
$$

where $i$ is the motor current, $R$ is the armature resistance, $L$ is the armature inductance, $K_{E}$ is the back-emf constant, $\omega_{M}$ is the motor angular velocity, $K_{T}$ is the torque constant, $J_{M}$ is the motor inertia, $\tau_{L_{M}}$ is the motor load torque, $\mu_{M}$ is the motor viscous friction constant and $\tau_{C_{M}}$ is the Coulomb motor friction torque (see also Fig. 4A). The model parameters were fitted using Multiple Regression Analysis of the form $y=a+b_{1} x_{1}+\ldots+b_{n} x_{n}$. A comparison of the model angular velocity $\omega_{M}$ with measurements for three different load levels is presented in Fig. 4B.

\section{B. Gearbox Model}

For the gearbox measurements it was not possible to measure the angular velocity at the gearbox shaft $\omega_{G}$. Hence, 
motor current $i$, gear ratio $N$ and input voltage $V$ were measured for different load torques $\tau_{L_{G}}$ and the model was again fitted using Multiple Regression Analysis. The model is equal to:

$$
\begin{aligned}
\omega_{G} & =\frac{\omega_{M}}{N} \\
\tau_{L_{G}} & =N \eta \tau_{L_{M}}-\tau_{C_{G}}-\omega_{G} \mu_{G}-J_{G} \frac{d \omega_{G}}{d t}
\end{aligned}
$$

where $J_{G}$ is the gearbox inertia, $\eta$ is the gearbox efficiency, $\tau_{C_{G}}$ is the Coulomb friction gearbox torque and $\mu_{G}$ is the gearbox viscous friction constant (see also Fig. 4A). The measured and simulated current of the model are presented in Fig. $4 \mathrm{C}$ for different $\tau_{L_{G}}$.

\section{State-space Model of DC Motor \& Gearbox}

For numerical integration a combined state-space representation of the DC motor and gearbox models was derived and integrated using a Forward-Euler integrator of the form $y(t+\triangle t)=y(t)+\dot{y}(t) \triangle t$. The Forward Euler integrator offers sufficient integration accuracy due to the slow system dynamics (see Fig. 4B,C) and, in the future, provides the integration performance required for real-time simulation of the more than 40 degrees of freedom of the ECCEROBOT torsos. After each integration step the kite line length is adapted in accordance to:

$$
L_{K}(t+\triangle t)=L_{K}(t)-\omega_{G} r \Delta t
$$

where $r$ is the gearbox shaft radius, and the resulting force $F_{L}$ is applied to the attachment points $\overrightarrow{A_{1}}$ and $\overrightarrow{A_{2}}$, respectively. When possible, the attachment points were positioned based on the laser scan data but simplifications were made to avoid the simulation of the pulleys (see Fig. 2).

\section{SENSOR Models}

As listed in Section II, the ECCEROBOT muscles are equipped with: (i) potentiometers, (ii) strain gauges and (iii) current sensors. These sensors were also modeled within Bullet Physics which makes it possible to transparently control the physical and simulated robot using the same controllers.

\section{A. Potentiometer Model}

Potentiometers attached to the gearbox shaft provide an absolute measure of the shaft position and hence of the kite line length. Provided there is an initial mapping between the kite length and the potentiometer value given, the current potentiometer value $S_{P}$ in degrees is equal to:

$$
S_{P}=360^{\circ} \cdot\left(\frac{L_{K}-L_{K_{0}}}{2 \pi r}\right)+S_{P_{0}}\left(L_{K_{0}}\right)
$$

where $S_{P_{0}}$ is the initial potentiometer position corresponding to the initial kite line length $L_{K_{0}}$.

\section{B. Force Sensor Model}

For the ECCEROBOTS custom force sensors had to be developed and manufactured as no off the shelf sensors were available that matched the size and performance requirements. In simulation, however, this sensor can easily be simulated by providing access to the ligament force $F_{L}$ which is recalculated during each simulation step.

\section{Current Sensor Model}

Current sensors have been included in the ECUs of the real robot to measure the magnitude of the current drawn by the DC motor [17]. This sensor has been also included in the simulation by providing the required interfaces to access the current $i$ of the DC motor model (see also Section V-A).

\section{Muscle Control}

A distributed, bio-inspired control architecture has been implemented for the ECCEROBOTS [17]. In this architecture, the individual muscles are controlled by dedicated ECUs with a control loop frequency of $500 \mathrm{~Hz}$. These ECUs were emulated by a software component using the provided CORBA interfaces of the CALIPER robot simulation environment (ROSE) [4]. Currently, a P controller for the kite line length and a state-space force controller for the ligament force $F_{L}$ are provided using the muscle and sensor models presented in Sections V and VI. Fig. 5 shows the performance of the kite line length controller exemplary for a pre-scripted elbow flexion when the shoulder joint was fixed. As mentioned in Section II, three muscles effect the elbow joint angle: (i) the bi-articular Biceps, (ii) the Brachialis, and (iii) the Triceps. Hence, only the reference and current gearbox shaft positions, as well as the forces of these muscles are plotted. The scripted movement starts at time $t_{s}$ with a new gearbox shaft reference position for the Brachialis, whereas the Biceps and Triceps follow with a delay of $250 \mathrm{~ms}$. At first the lever arms of the Biceps and Brachialis are extremely short due to the almost extended elbow joint $(-0.57 \mathrm{rad})$ which results in the high peak of the Biceps force at marker $m_{1}$. However, due to the compliant actuation, no trajectory planning is required and the timing of the individual control signals is not as important as for traditional robots. This can be seen at $m_{2}$ where the Biceps and Triceps are pulling against each other. This is an elegant example of Morphological Computation [20] as the exact timing of actuation commands and the resulting trajectory is handled by the skeleton and the tendon-driven actuators of the test rig. Finally the gearbox positions, the muscle forces and the joint angle reach a steady state $\left(m_{3}\right)$.

\section{CONCLUSIONS AND FUTURE WORKS}

\section{A. Conclusions}

We proposed to use physics-based simulation engines for the simulation of the highly complex dynamics of tendon-driven robots and derived a detailed physics-based model of the skeleton, as well as of the passive ligament and active muscle components of an anthropomimetic robot. Due to the handcrafted manufacturing of the skeleton, we had to elaborately 


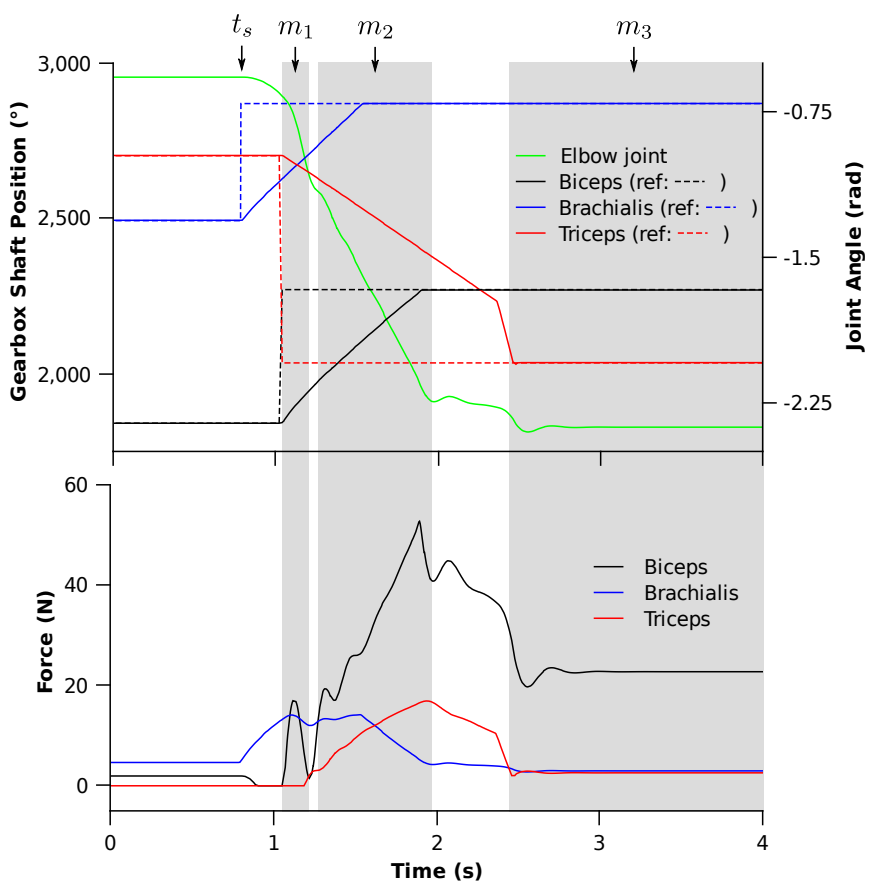

Fig. 5. Elbow Movement Example. Top: Three muscles affect the elbow joint angle: (i) Biceps, (ii) Brachialis, and (iii) Triceps. The gearbox spindle position is controlled using a $\mathrm{P}$ controller. By setting new reference values, the muscles exert force on the bones of the robot and the elbow joint angle changes accordingly. Bottom: Force of the Biceps, the Brachialis, and Triceps during the movement.

reverse-engineer the structure using true to scale photographs and laser scans. Furthermore, the peculiar actuation concept of this class of robot required custom extensions to be implemented for the physics engine, namely models of the DC motor, the gearbox, and of the passive kite line and shock cord components. Additionally, Coulomb, as well as viscous friction support had to be added for the used constraint types to simulate the damping properties of the test rig. Furthermore, low-level muscle controllers were implemented which provide a high-level interface for evoking actuation commands. Finally, the integration of the individual sub-models and the feasibility of the physics-based approach was demonstrated by executing a limb movement that comprises the simultaneous control of three muscles.

\section{B. Future Works}

A detailed analysis of the performance of the complete, integrated simulation model is still lacking. Hence, we will develop an external sensing system to acquire the joint angles of the physical test rig and that will allow us to quantify and subsequently calibrate the performance of the simulated robot. Furthermore, based on the lessons learned from modeling the test rig, we will derive a physics-based model of the highly complex ECCE-I successor ECCE-II. Fortunately, only the modeling of the skeleton is required as all other tools (joint friction, ligament and muscle models) have been already developed and validated using the small-scale setup of the test rig.

\section{ACKNOWLEDGMENTS}

We thank Cristiano Alessandro (Artificial Intelligence Laboratory, Department of Informatics, University of Zurich, Switzerland) for characterizing the properties of the shock cord.

The research leading to these results has received funding from the European Community's Seventh Framework Programme FP7/2007-2013 Challenge 2- Cognitive Systems, Interaction, Robotics - under grant agreement no. 231864- ECCEROBOT.

\section{REFERENCES}

[1] H. Kino, S. Kikuchi, T. Yahiro, and K. Tahara, "Basic study of biarticular muscle's effect on muscular internal force control based on physiological hypotheses," in Proc. IEEE International Conference on Robotics and Automation ICRA '09, 2009, pp. 4195-4200.

[2] S. C. Jacobsen, H. Ko, E. K. Iversen, and C. C. Davis, "Antagonistic control of a tendon driven manipulator," in Proc. Conf. IEEE Int Robotics and Automation, 1989, pp. 1334-1339.

[3] M. Kawato, "Internal models for motor control and trajectory planning," Current Opinion in Neurobiology, vol. 9, no. 6, pp. 718-727, 1999.

[4] S. Wittmeier, M. Jäntsch, K. Dalamagkidis, M. Rickert, H. G. Marques, and A. Knoll, "Caliper: A Universal Robot Simulation Framework for Tendon-Driven Robots," in Proc. IEEE/RSJ International Conference on Intelligent Robots and Systems (IROS), 2011, accepted.

[5] Embodied Cognition In A Compliantly Engineered Robot (ECCEROBOT). [Online]. Available: http://www.eccerobot.eu

[6] S. Wittmeier, G. Song, J. Duffin, and C.-S. Poon, "Pacemakers handshake synchronization mechanism of mammalian respiratory rhythmogenesis." Proc Natl Acad Sci USA, vol. 105, no. 46, pp. 18000-18 005, Nov 2008.

[7] W. Leontief, F. Duchin, and D. B. Szyld, "New Approaches in Economic Analysis," Science, vol. 228, no. 4698, pp. 419-422, 1985.

[8] R. Blumhardt, "Fem - crash simulation and optimisation," International Journal of Vehicle Design, vol. 26, no. 4, pp. 331-347, Jan. 2001.

[9] J.-J. E. Slotine and W. Li, "On the Adaptive Control of Robot Manipulators," The International Journal of Robotics Research, vol. 6, no. 3, pp. 49-59, 1987.

[10] NVIDIA Physx. NVIDIA. [Online]. Available: http://www.nvidia.com/object/physx_new.html

[11] E. Coumans. Bullet Physics Library. Sony Computer Entertainment. [Online]. Available: http://www.bulletphysics.com

[12] O. Holland and R. Knight, "The anthropomimetic principle," in Proceedings of the AISBO6 Symposium on Biologically Inspired Robotics, 2006.

[13] H. Marques, M. Jäntsch, S. Wittmeier, O. Holland, C. Alessandro, A. Diamond, M. Lungarella, and R. Knight, "Ecce1: The first of a series of anthropomimetic musculoskeletal upper torsos," in Humanoid Robots (Humanoids), 2010 10th IEEE-RAS International Conference on, 2010, pp. $391-396$.

[14] Y. Sakagami, R. Watanabe, C. Aoyama, S. Matsunaga, N. Higaki, and K. Fujimura, "The intelligent asimo: system overview and integration," in Intelligent Robots and Systems, 2002. IEEE/RSJ International Conference on, vol. 3, 2002, pp. $2478-2483$ vol.3.

[15] K. Kaneko, K. Harada, F. Kanehiro, G. Miyamori, and K. Akachi, "Humanoid robot hrp-3," in Intelligent Robots and Systems, 2008. IROS 2008. IEEE/RSJ International Conference on, 2008, pp. $2471-2478$.

[16] E. R. Kandel, J. Schwartz, and T. M. Jessell, Principles of Neural Science, 4th ed. Mc Graw Hill, 2000.

[17] M. Jäntsch, S. Wittmeier, and A. Knoll, "Distributed Control for an Anthropomimetic Robot," in Intelligent Robots and Systems (IROS), 2010 IEEE/RSJ International Conference on, 2010, pp. 5466 -5471.

[18] K. Erleben, "Stable, robust, and versatile multibody dynamics animation," Ph.D. dissertation, University of Copenhagen, Denmark, November 2004.

[19] J. Chiasson, Modeling and High Performance Control of Electric Machines. Wiley-IEEE Press, 2005.

[20] R. Pfeifer and G. Gómez, "Morphological Computation Connecting Brain, Body, and Environment," in Creating Brain-Like Intelligence, ser. Lecture Notes in Computer Science, B. Sendhoff, E. Krner, O. Sporns, H. Ritter, and K. Doya, Eds. Springer Berlin / Heidelberg, 2009, vol. 5436, pp. 66-83. 